\title{
Complications and Survival in Esophagojejunostomy after Total Gastrectomy; A Comparision of Stapler and Manual Suturing
}

\author{
Akhavanmoghadam J. ${ }^{1}$ MD, Mohebbi H.A. ${ }^{1}$ MD, Taheri S.M.* ${ }^{*}$, Nikkhah.$^{2}$ BSc \\ *Surgery Department, Medicine Faculty, Baqiytallah University of Medical Science, Tehran, Iran \\ ${ }^{1}$ Surgery Department, Medicine Faculty, Baqiytallah University of Medical Science, Tehran, Iran \\ ${ }^{2}$ Midwifery Department, Nursing and Midwifery Faculty, Shahid Beheshti university of medical science, \\ Tehran,Iran
}

\begin{abstract}
Aims: Gastric cancer is the most common cancer in men and the third most common cancer in women after breast cancer and colon. This study aimed to compare the complications and survival of two manual and suturing and suturing with stapler in esophagojejunostomy suturing followed by total gastrectomy.

Materials \& Methods: In this historical cohort study, 116 patients requiring total Gastrectomy were studied with proximal Gastric and Cardia cancer referred to a hospital with census method. Patients were studied by age, gender, type of surgery, leakage and other complications and one-year survival rates during hospitalization and at least 12 months after surgery. Data analyzed by SPSS 20 software and Chi-square, Independent-T tests, Kaplan-Meier and Cox regression.

Findings: Suturing in 40 patients (34.5\%) was manually and in 76 patients $(65.5 \%)$ was with stapler. In manual suturing, 6 (15\%) and in stapler suturing 2 $(2.6 \%)$ leakage happened, which was statistically different $(\mathrm{p}=0.18)$. Mean operation time was $273.9 \pm 50.7 \mathrm{~min}$ in manual suturing and was $252.3 \pm 65.8$ in stapler suturing which was statistically different $(\mathrm{p}=0.25)$. In manual suturing 8 $(20 \%)$ and in stapler suturing 4 (5.3\%) deaths occurred which was statistically different $(\mathrm{p}=0.009)$. One-year survival rate was $31 \%$ and median survival rate was 15 months. Age and stage of disease had the highest correlation with survival rate.

Conclusion: Due to the lower leakage rate and mortality after surgery, manual suturing is more effective than suturing with stapler.

\section{Keywords}

Surgical Staplers [http://www.ncbi.nlm.nih.gov/mesh/68013528];

Anastomosis, Surgical [http://www.ncbi.nlm.nih.gov/mesh/68000714];

Sutures [http://www.ncbi.nlm.nih.gov/mesh/68013537];

Stomach [http://www.ncbi.nlm.nih.gov/mesh/68013270]
\end{abstract}

* Corresponding Author

Tel: +982177786673

Fax: +982177786673

Address: Unit 33, West First Floor, No. 348, East 212 Street, Tehranpars, Tehran, Iran

tahermojtaba@gmail.com

Received: January 11, $2014 \quad$ Accepted: July 28, 2014 ePublished: February 19, 2015 


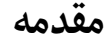

معده قسمتى از دستخاه كَارش است كه در حد واسط مرى و وارو

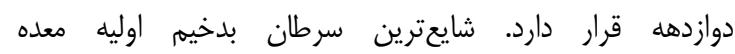

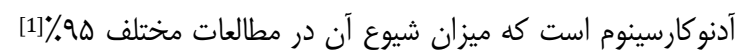

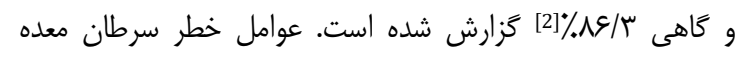

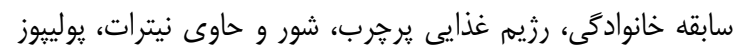

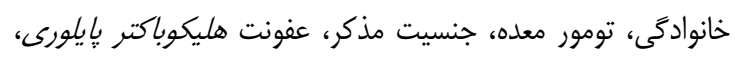

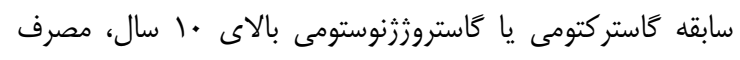

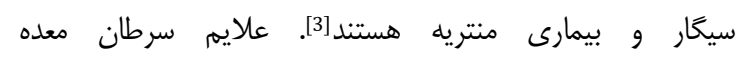

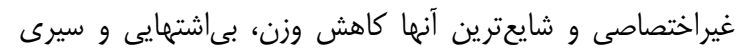

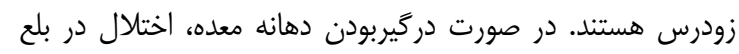

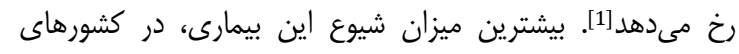

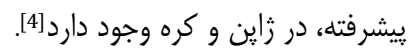

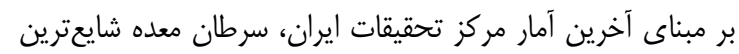

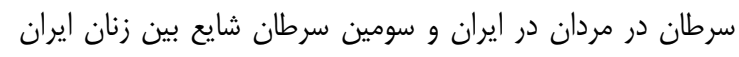

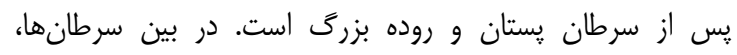

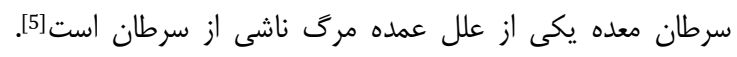

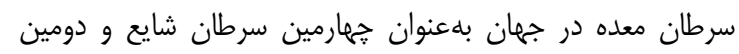

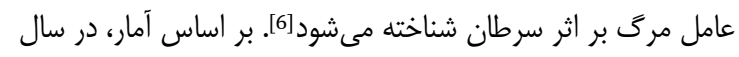

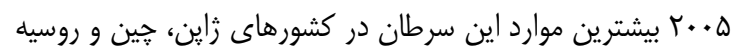

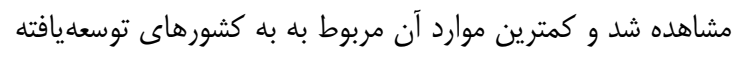

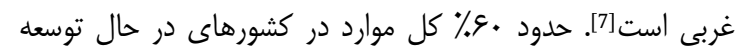

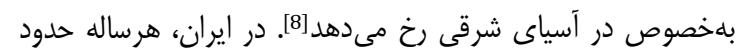

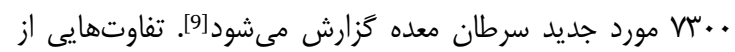

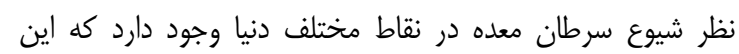

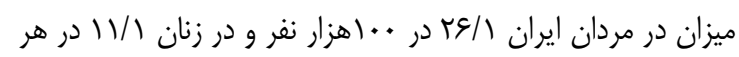

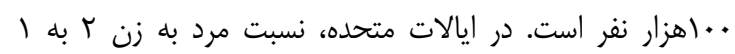

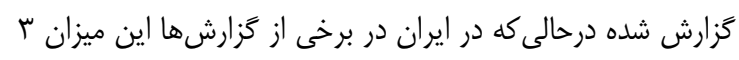

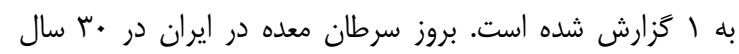
كذشته افز ايش داشته است [10].

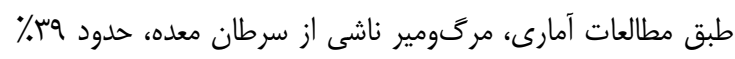

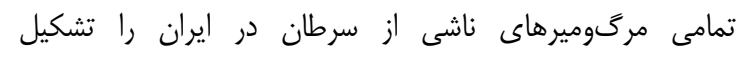

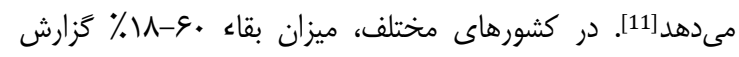

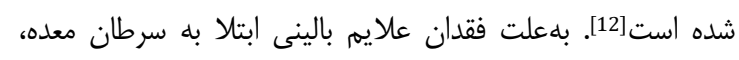

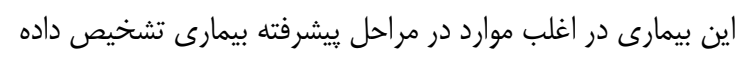

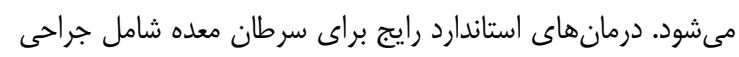

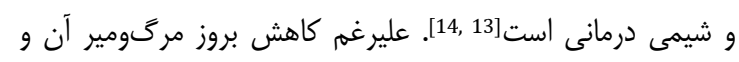

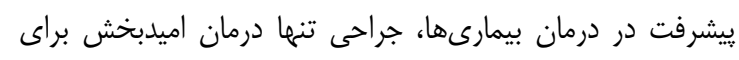

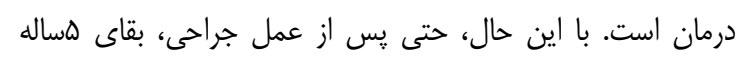

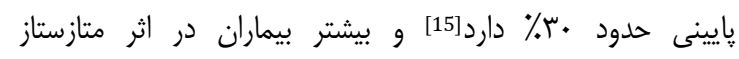

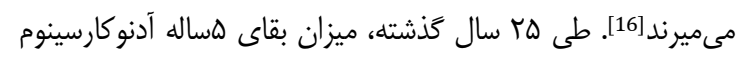

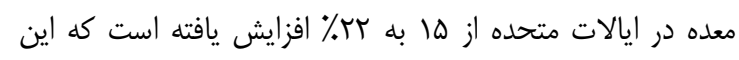

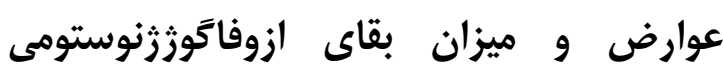
بلهدنبال كَاستركتومى توتال؛ مقايسه دو دور روش بخيه دستى و منگَنه جراحى

جمال اخوانمقدم MD

كَروه جراحى عمومى، دانشكده يزشكى، دانشكاه علوم يزشكى بقيها...(كجا، تهران،

ايران

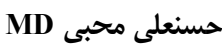

كروه جراحى عمومى، دانشكده يزشكى، دانشكاه علوم يزشكى بقيها.... (عج)، تهران،

ايران

MD " سيدمجتبى طاهرى

كروه جراحى عمومى، دانشكده يزشكى، دانشكاه علوم يزشكى بقيها.... (عج)، تهران،

ايران

سميه نيكخواه BSc

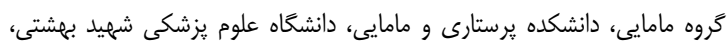

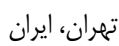

جكيده

اهداف: سرطان معده شايعترين سرطان در مردان در ايران و سومين

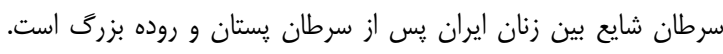

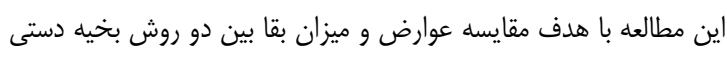

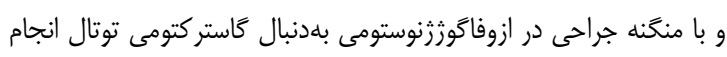

شد.

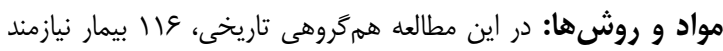

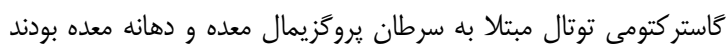

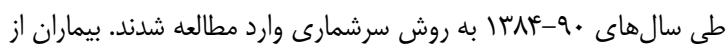

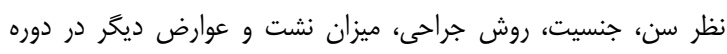

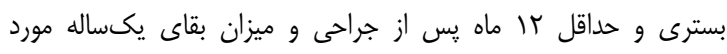

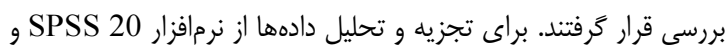

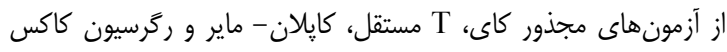

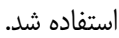

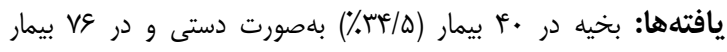

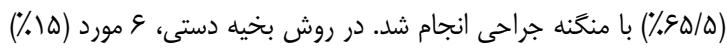

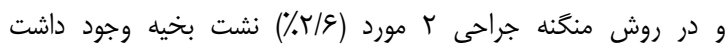

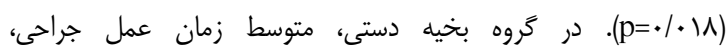

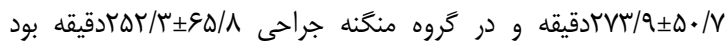

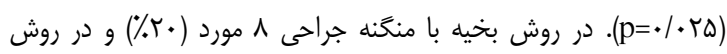

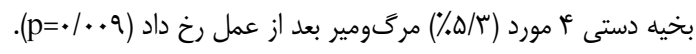

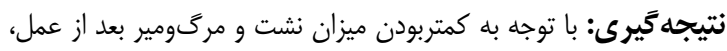

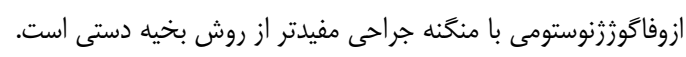

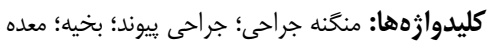

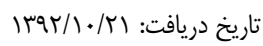

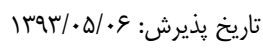
tahermojtaba@gmail.com : تويسنده مسئول:

دوره •r، شماره f، زمستان بوجا

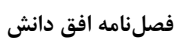




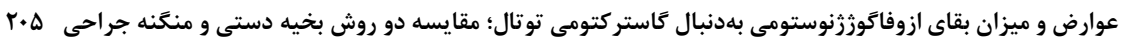

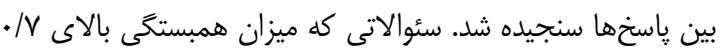
داشتند مورد قبول واقع شدند.

با بيماران ترخيصشده از بيمارستان تماس كرفته شد و در مر مورد

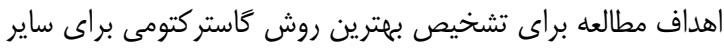
بيماران و بررسى عوارض جراحى در خود اين بيماران توضيح داده

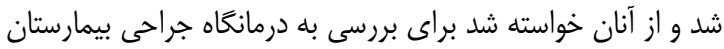
مراجعه كنند و در صورت فوت بيمار از بستخان درجه يكى خواسته بهاس

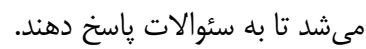

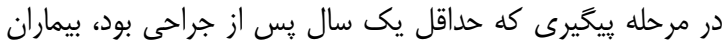

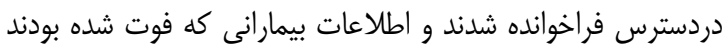

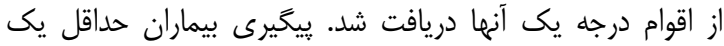

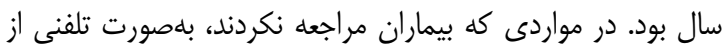

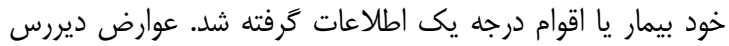
شامل تنگى محل بخيه، فتق شكافى و درمانهاى تكميلى و ميزان بقاى بيماران ييخيرى و ثبت شد.

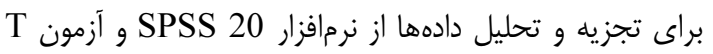
مستقل براى مقايسه مشخصات جمعيتشناختى و نوع تومور و و وران

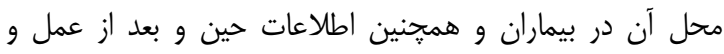
عوارض مربوطه در بيمارستان بين دو گروه و براى بررسى بقا از آزمون كايلان - ماير و رَرسيون كاكس استفاده شد.

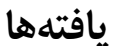

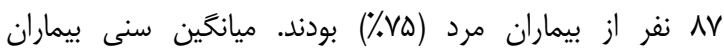

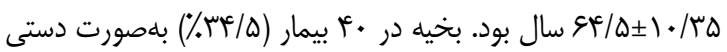

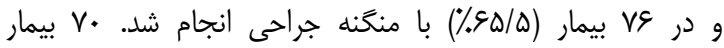

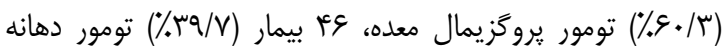

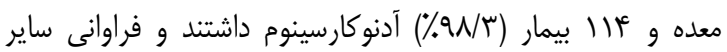

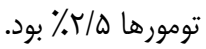

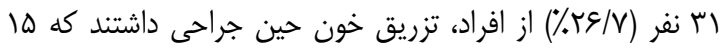

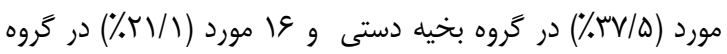

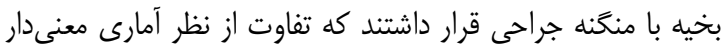

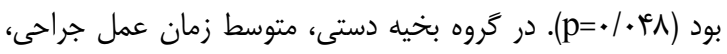

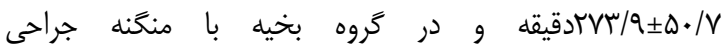
1/A

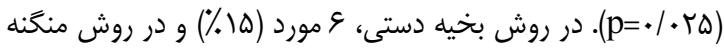

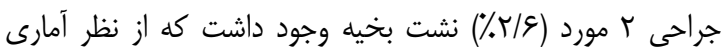

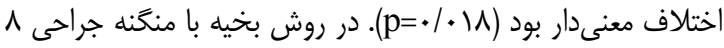

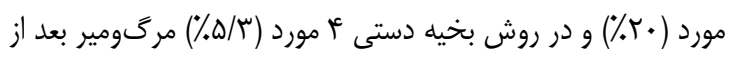

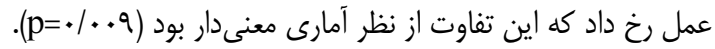

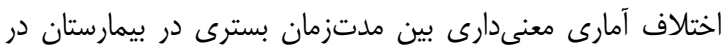

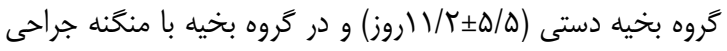

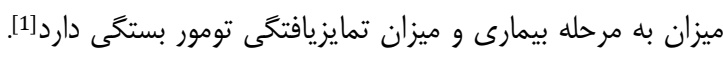

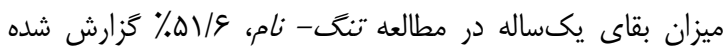

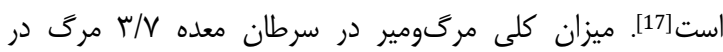

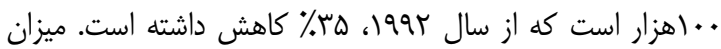

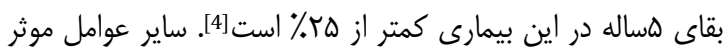
بر ييشآَّمى، جنسيت، سن، اندازه تومور، عمق تومور و محل اوليه تومور هستند[1].

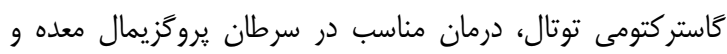

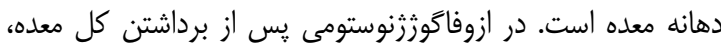

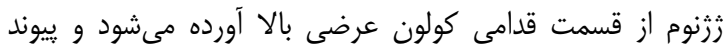

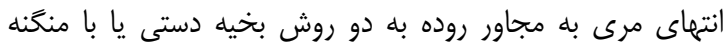

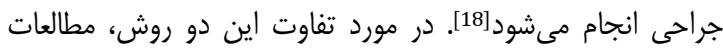
مختلف و كزارشهاى متعددى در دنيا انجام شده است. در اكثر

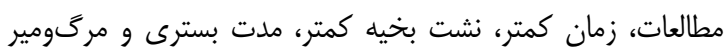

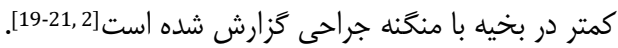

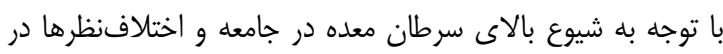

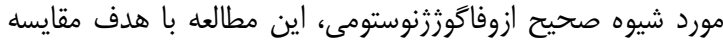
ميزان عوارض و بقا بين دو روش بخيه دستى و بخيه با با منكَنه جراحى در ازوفاكوزثنوستومى بلدنبال كاستر كتومى توتال انجام شد.

\section{مواد و روشها}

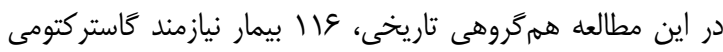

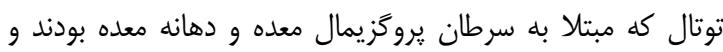

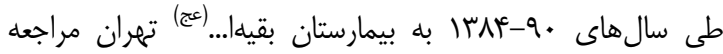

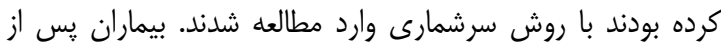

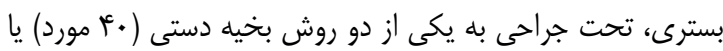

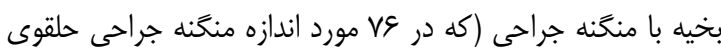

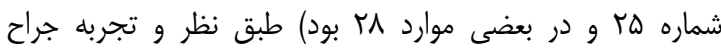

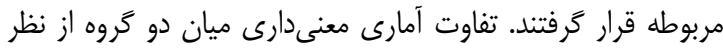

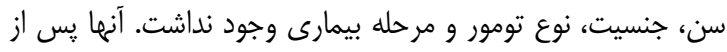

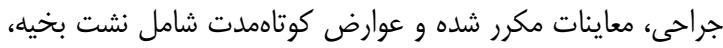

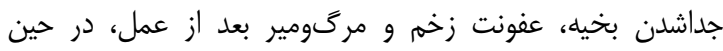
بسترى، تا ترخيص بررسى و ثبت شدات زمات

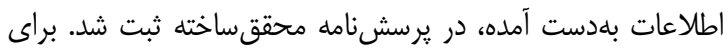
تعيين اعتبار يرسشنامه، از روش اعتبار محتوا استفاده شد.

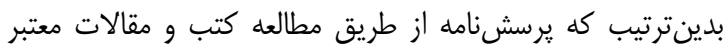

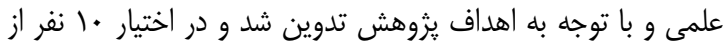

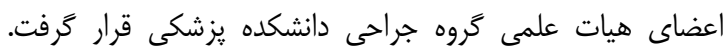

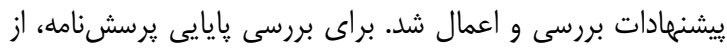

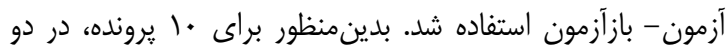

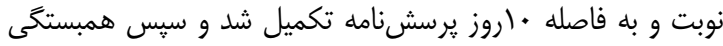




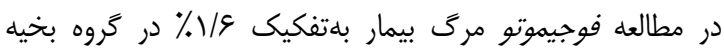

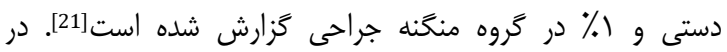

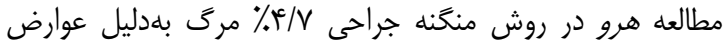

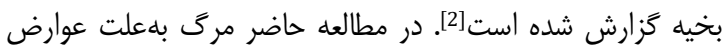
نسبت به ساير مطالعات بيشتر بود كه مىتواند بهعلت بيشتربودن نشت نسبت به ساير مطالعات باشد.

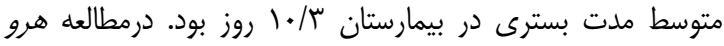

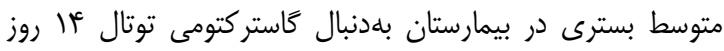
گَزارش شده است[2]. در مطالعهاى ديخر مدت بسترى درى در بيمارستان

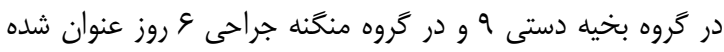
است[19]. بيشتربودن مدت بسترى دستى در بيمارستان در مطالعه حاضر

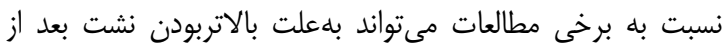
عمل در بيماران و ديرتر شروعكردن تغذيه دهانى در بيماران بسترى در بخش جراحى بهدنبال كاستر كتومى توتال باشد.

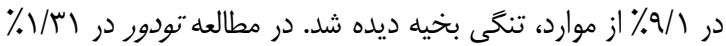

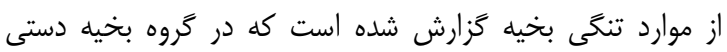

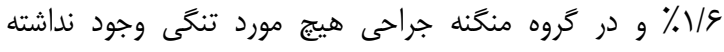

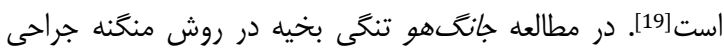

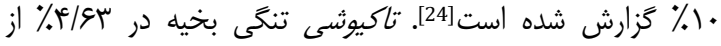

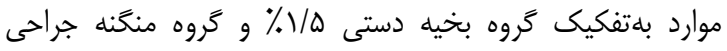

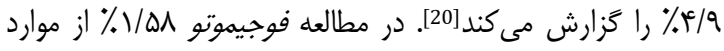

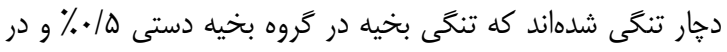

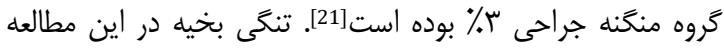

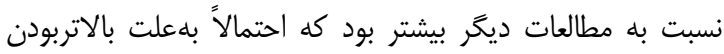
ميزان نشت بخيه است.

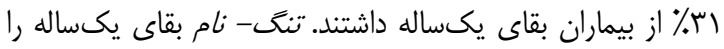

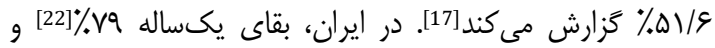

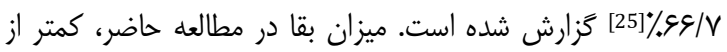

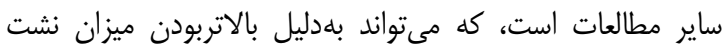
بخيه بعد از عمل باشد. در اين مطالعه، سن و مرحله بيمارى

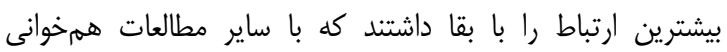

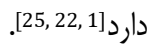
از آن جايى كه تكنيك انجامشده روى بيماران منتخب كاستر كتومى قبلاً انجام شده، امكان كنترل عواملى نظير بيمارىهاى همراه، وضع تغذيه، سابقه كموراديوترايى و وضعيت سيستم ايمنى در دو گروه وجود نداشت. برنامهريزى براى ييخيرى دقيق بيماران و ثبت ونيت

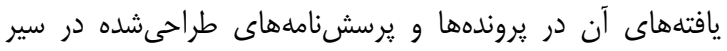
درمان؛ برقرارى ارتباط مناسب و منسجم و هدفدار بإ بيماران و و

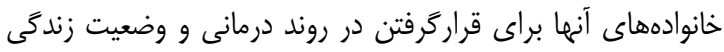

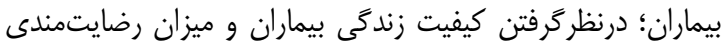

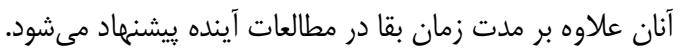

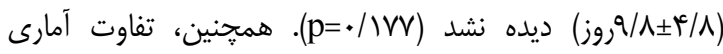

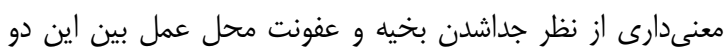

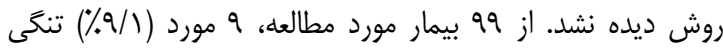

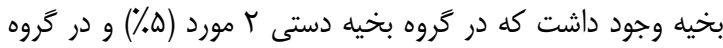
بخيه با منگُنه جراحى V مورد (T/9\%) بود، ولى اين اختلاف از نظر

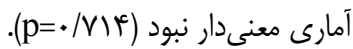

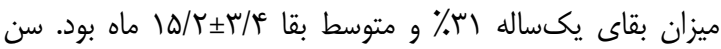

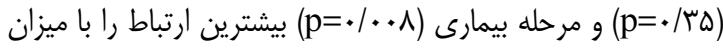
بقا داشتند.

\section{بحث}

سرطان معده، شايعترين سرطان در مردان و سومين سرطان شايع

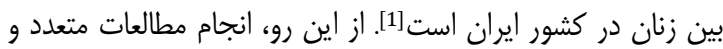
فراگير در اين زمينه و بحثهاى كارشناسى براى روشهاى درمانى

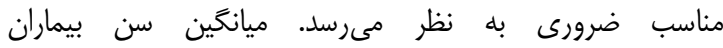

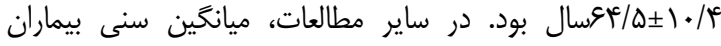

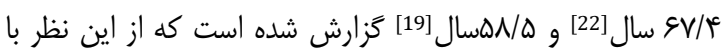
ساير مطالعات همخوانى دارد. در هيجكدام از دو گروه موردى از فتق شكافى وجود نداشت. فراوانى سرطان معده در مردان VD٪ و س برابر فراوانى اين سرطان

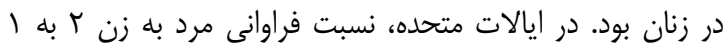
گزارش شده است[1]. در ايران نيز، در برخى از زَارشها اين ميزان

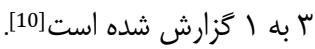

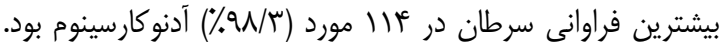

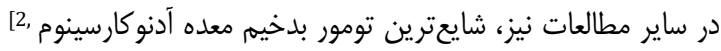

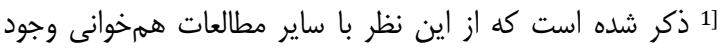

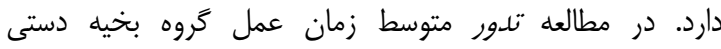

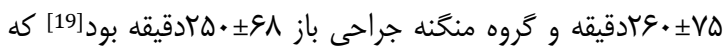

با مطالعه انجامشده همخوانى دارد.

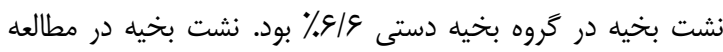

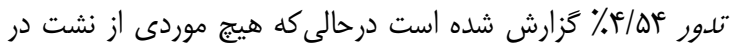

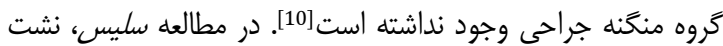

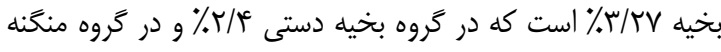

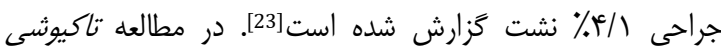
ميزان نشت بخيه سس/\%/ است كه س/س\% در گروه بخيه دستى و

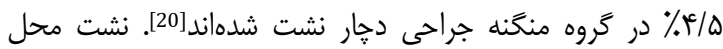
بخيه در مطالعه اخير نسبت به مطالعات ديخر بيشتر بود كه احتمالاً بلهدليل بيشتربودن محل تومور در دهانه معده است. از طرفى، انجام

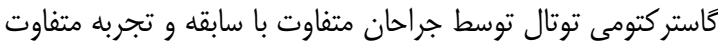
مىتواند در اين امر نقش بلهز ايیى داشته باشد. 


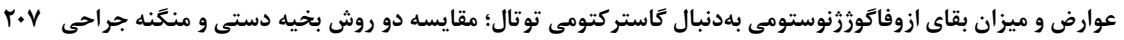

11- Yazdanbod A, Arshi S, Derakhshan MH, Sadjadi AR, Malekzadeh R. Gastric cardia cancer; the most common type of upper gastrointestinal cancer in Ardabil, Iran: An endoscopy clinic experience. Arch Irn Med. 2001:4(2):76-9. [Persian]

12- Jain VK, Cunningham D, Rao S. Chemotherapy for operable gastric cancer: current perspectives. Indian J Surg Oncol. 2011;2(4):334-42.

13- Lang SA, Gaumann A, Koehl GE, Seidel U, Bataille F, Klein D, et al. Mammalian target of rapamycin is activated in human gastric cancer and serves as a target for therapy in an experimental model. Int J Cancer. 2007;120(8):1803-10.

14- Wang F, Li T, Zhang B, Li H, Wu Q, Yang L, et al. MicroRNA- 19a/b regulates multidrug resistance in human gastric cancer cells by targeting PTEN. Biochem Biophys Res Commun. 2013;434(3):688-94.

15- Dicken BJ, Bigam DL, Cass C, Mackey JR, Joy AA, Hamilton SM. Gastric adenocarcinoma: review and considerations for future directions. Ann Surg 2005;241(1):27-39.

16- Dragovich T, Campen C. Anti-EGFRTargeted Therapy for Esophageal and Gastric Cancers: An Evolving Concept. J Oncol. 2009;2009:804108.

17- Thong-Ngam D, Tangkijvanich P, Mahachai V, Kullavanijaya P. Current status of gastric cancer in Thai patients. J Med Assoc Thai. 2001;84(4):475-82.

18- Zinner MJ, Ashley SW. Maingot's Abdominal Operations. $12^{\text {th }}$ ed. China: McGraw-Hill Companies; 2013.

19- Tudor S, Anton A, Purnichescu-Purtan R. Stapled esophagojejunal anastomoses: particular aspects of minimally invasive surgery and comparison with manual anastomoses: A single team experience. Chirurgia (Bucur). 2012;107(2):169-73.

20- Takeyoshi I, Ohwada S, Ogawa T, Kawashima Y, Ohya $\mathrm{T}$, Kawate $\mathrm{S}$, et al. Esophageal anastomosis following gastrectomy for gastric cancer: Comparison of hand-sewn and stapling technique. Hepatogastroenterology. 2000;47(34):1026-9.

21- Fujimoto S, Takahashi M, Endoh F, Takai M, Kobayashi $\mathrm{K}$, Kiuchi S, et al. Stapled or manual suturing in esophagojejunostomy after total gastrectomy: A comparison of outcome in 379 patients. Am J Surg. 1991;162(3):256-9. 22- Maroufizadeh S, Hajizadeh E, Baghestani A, Fatemi S. Prognostic factor for patients with gastric cancer using the Aalen's additive hazards model. Koomesh. 2011;13(1):1206. [Persian]

23- Celis J, Ruiz E, Berrospi F, Payet E. Mechanical versus manual suture in the jejunal esophageal anastomosis after total gastrectomy in gastric cancer. Rev Gastroenterol Peru. 2001;21(4):271-5.

24- Shim JH, Oh SI, Yoo HM, Jeon HM, Park CH, Song KY. Short-term outcome of laparascopic versus open total gastrectomy: A matched-cohort study. Am J Surg. 2013;206(3):346-51.

25- Zeraati H, Mahmoodi M, Kazemnejad MK, Mohagheghi MA. Prospective survival in gastric cancer patients and its related factors. J School Public Health Inst Public Health Res. 2005;3(4):1-2. [Persian]

$$
\begin{aligned}
& \text { نتيجهد كيرى } \\
& \text { با توجه به كمتربودن ميزان نشت و مركَومير بعد از عمل، } \\
& \text { ازوفاگوثزنوستومى با منگَنه جراحى مفيدتر از روش بخيه دستى } \\
& \text { است. } \\
& \text { تشكر و قدردانى: بدينوسيله از همكاران و اساتيد محترم گروه } \\
& \text { جراحى تشكر و قدردانى مىشود. اين مقاله منتج از ياياننامه } \\
& \text { دكتراى تخصصى جراحى عمومى و مصوب در معاونت تحقيقات و ونى } \\
& \text { فنّاورى دانشخاه علوم يزشكى بقيها... (عج) است. } \\
& \text { تاييديه اخلاقى: موردى از طرف نويسندكان گزارش نشده است. } \\
& \text { تعارض منافع: موردى از طرف نويسندگان گزارش نشده است. } \\
& \text { منابع مالى: كليه هزينهها بر عهلده محقق بوده است. }
\end{aligned}
$$

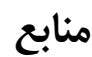

1- Brudicardi F, Anderson D, Billiar T, Dunn D, Hunter J, Matthews J. Schwartz's Principles of Surgery. $10^{\text {th }} \mathrm{ed}$. New York: McGraw-Hill Professional; 2014.

2- Herve J, Simoens Ch, Smets D, Thill V, Mendes Da Costa P. Mechanical esophageal anastomosis: Retrospective study of 56 patients. Jurnalul de Chirurgie Iaşi. $2009 ; 5(1): 34-44$.

3- Fox JG, Wang TC. Inflammation, atrophy, and gastric cancer. J Clin Invest. 2007;117(1):60-9.

4- Townsend CM, Beauchamp RD, Evers BM, Mattox KL. Sabiston Textbook of Surgery. $18^{\text {th }}$ ed. Philadelphia: Elsevier; 2008

5- Heise K, Bertran E, Andia ME, Ferreccio C. Incidence and survival of stomach cancer in a high-risk population of Chile. World J Gastroenterol. 2009;15(15):1854-1862.

6- Jemal A, Bray F, Center MM, Ferlay J, Ward E, Forman D. Global cancer statistics. CA Cancer J Clin. 2011;61(2):69-90

7- Inoue M, Tsugane S. Epidemiology of gastric cancer in Japan. Postgrad Med J. 2005;81(957):419-24.

8- Jemal A, Siegel R, Ward E, Murray T, Xu J, Smigal C, et al. Cancer statistics, 2006. CA Cancer J Clin. 2006;56(2):106-30.

9- Movahedi M, Afsharfard A, Moradi A, Nasermoaddeli A, Khoshnevis J, Fattahi F, et al. Survival rate of gastric cancer in Iran. J Res Med Sci. 2009;14(6):367-73. [Persian] 10- Sajadi A, Nouraei M, Mohagheghi MA, MousaviJarrahi A, Malekezadeh R, Parkin DM. Cancer Occurrence in Iran in 2002, an internationalperspective. Asian Pac J Cancer Prev. 2005;6(3):359-63. [Persian] 